Reflexiones contables (Cúcuta)

102

https://doi.org/10.22463/26655543.2991

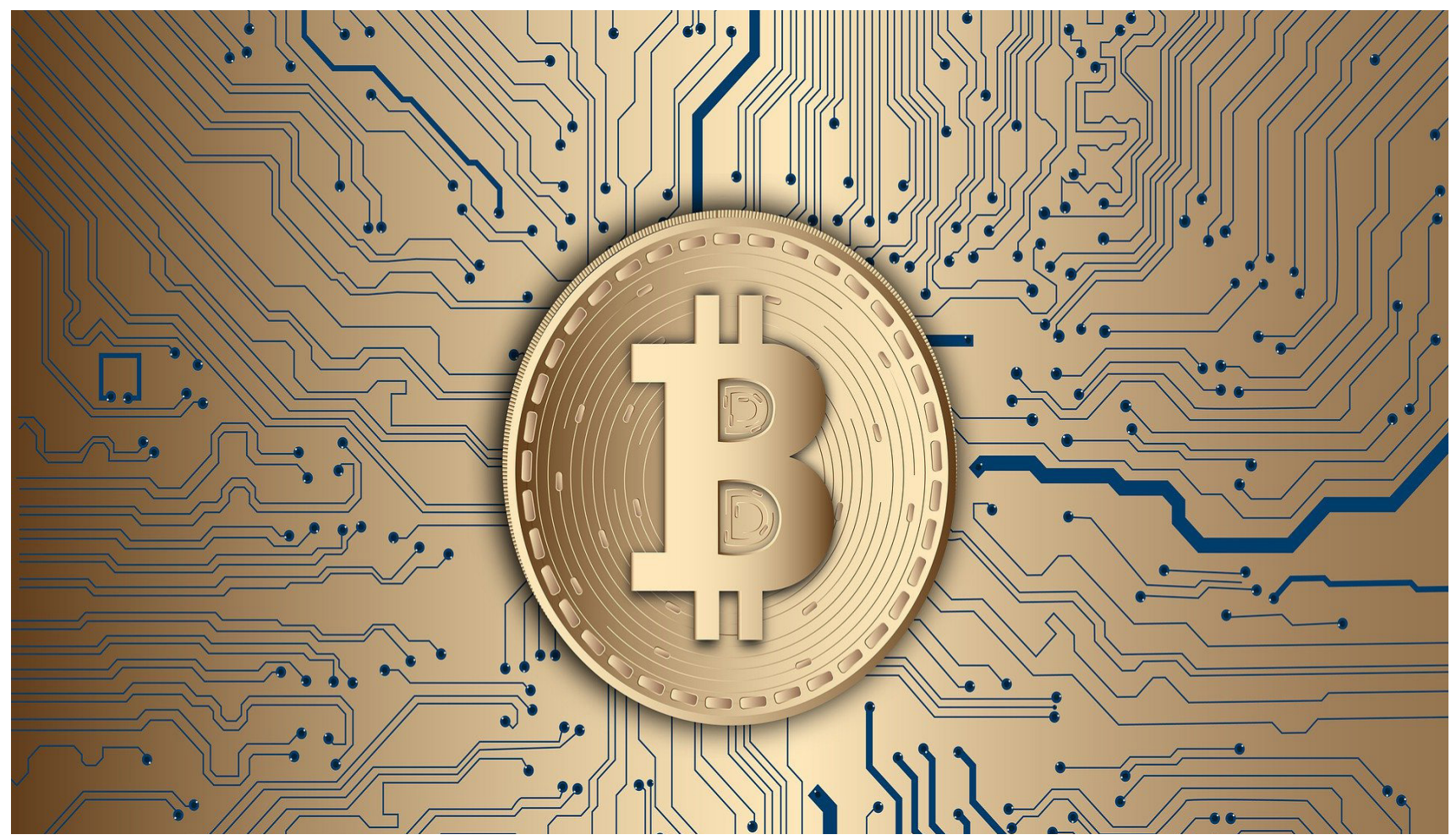

\title{
La confiabilidad en las monedas digitales como inversión práctica en la economía del futuro
}

\section{Reliability in Digital Currencies as a Practical Investment in the Economy of the Future}

Julio Cesar González-Millan

Estudiante del Programa de Contaduría Pública, Universidad Francisco de Paula Santander, Cúcuta, Colombia

Luis Fernando Guardiola-Plazas

Magister en Gerencia de Empresas mención Finanzas, luisfernandogp@ufps.edu.co, Universidad Francisco de Paula Santander, Cúcuta, Colombia 
Cómo citar: González-Millan, J.C., Guardiola-Plazas, L.F. (2019). La confiabilidad en las monedas digitales como inversión práctica en la economía del futuro. Reflexiones contables (Cúcuta), 2 (2), 102-114.

\section{Resumen}

En el mundo de la era tecnológica, las monedas digitales están marcando el inicio de una revolución industrial que podría llamarse revolución de las criptomonedas, cada país le da su valor sobre la economía funcional que posee, y tiene una moneda para comercializar a través de la tecnología digital, lo que presumiblemente hasta hoy es una evolución de monedas intangibles, y está desplazando los billetes, pero que también se están imprimiendo billetes sobre criptomonedas, lo que quiere decir que es dinero efectivo, seguro y práctico. En el presente trabajo se realiza proponiendo como objetivo general: Determinar la confiabilidad en las monedas digitales como inversión práctica en la economía del futuro. Se realizó una investigación tipo documental y descriptiva, de análisis, basadas en revistas, fuentes de internet, informes, y libros respecto al tema. Se concluye que la confiabilidad de la moneda es alarmante, respecto a la cantidad de inversiones que están haciendo las personas y los comercios con esta revolución de economía digital, pero que el dólar es difícil que se desplace, porque casi todos los países del mundo comercializan sus productos con esta divisa, y Colombia cuenta con proyecciones de cambiar su moneda colombiana, dada a conocer la criptomoneda Triskel, aunque lo difícil no es crearla sino mantener su poder de convencimiento.

Palabras clave: Criptomonedas, Confiabilidad, Inversión, Economía

\section{Abstract}

In the world of the technological era, digital coins are marking the beginning of an industrial revolution that could be called the crypto coin revolution, each country gives its value over the functional economy it has, and has a currency to market through digital technology, which presumably until today is an evolution of intangible coins, and is displacing banknotes, but which are also being printed on crypto coin, which means that it is effective, safe and practical money. In the present work it is carried out proposing as a general objective: To determine the reliability of digital coins as a practical investment in the economy of the future. A documentary and descriptive research was carried out, based on magazines, internet sources, reports and books on the subject. It is concluded that the reliability of the currency is alarming, with respect to the amount of investments that people and businesses are making with this digital economy revolution, but that the dollar is difficult to move, because almost all countries in the world market their products with this currency, and Colombia has projections of changing its currency, released the Triskel crypto-currency, although the difficult thing is not to create it but to maintain its power of conviction.

Keywords: Cryptocurrencies, Reliability, Investment, Economy 


\section{4}

\section{Introducción}

El crecimiento económico desde las grandes economías de occidente en las últimas tres décadas del siglo pasado ha hecho que grandes potencias tengan problemas para los manejos de los créditos; y el flujo económico en la impresión de billetes ha llevado a los países al endeudamiento, lo cual es el causante de la caída económica; pero en muchas partes ha crecido la población, lo que ha reducido el empleo, la calidad de vida para países subdesarrollados. (Barrutia, Urquizo, \& Acevedo, 2019)

En este orden de ideas y a raíz de las tecnologías y la globalización se habla de otros términos de la economía, (Fernando \& González, 2018), el papel moneda ya no es eficiente, está siendo reemplazado paulatinamente para algunos países, (Jiménez, 2020); hoy se está hablando de un mundo mediático con respecto al dinero, intangible que sólo se ve valorizado en una tarjeta plástica y coge valor para el que realice mayores transacciones bancarias; y que el usuario solo necesita poseer un teléfono de última gama para acceder a ellos. (Caballero, 2019)

Colombia por esta parte, ha querido instaurar la primera moneda digital, llamada Triskel, hecha por la empresa colomboamericana Fenix Premiun, además respaldada por finca raíz, quien asegura su fundador que posee respaldo a nivel internacional, y cuenta con un amplio registro de transacciones apoyados en las empresas colombianas, brindando la confiabilidad que las transacciones sean de confiabilidad; pues cada una de ellas tiene funcionalidades diferentes; por ejemplo el software del protocolo del bitcoin (Mecheba, 2016), que este abierto para que cualquier persona pueda copiar y crear su propia criptomoneda, se crearían tantas monedas que en mismo país estarían circulando varias de ellas, lo cual no daría credibilidad a la escogencia, se preferiría la divisa física más segura: el dólar. (Restrepo \& Ocampo, 2019); (Melguizo, 2020);

Asimismo, se establece la interrogante, si ¿Serán confiables las monedas digitales para las inversiones particulares o empresariales en Colombia? ¿Acaso se pretende con ellas el desplazamiento del dólar? ¿Esta revolución digital, que está desplazando los billetes, se puede convertir en papel moneda más luego y qué efecto tendría?; con estas interrogantes se pretende realizar la siguiente investigación, Como objetivo general se plantea: Determinar la confiabilidad en las monedas digitales como inversión práctica en la economía del futuro; y como objetivos específicos: a) Describir el comportamiento económico en materia de inversiones en Colombia; b) Analizar si las operaciones cambiarias pretenden el desplazamiento del dólar; c) Determinar si las criptomonedas son confiables para las inversiones económicas de las empresas colombianas.

\section{Desarrollo}

A raíz de las economías virtuales del futuro están hablando de criptomonedas, que son monedas inteligentes creadas a través de un software, o dinero intangible, que la mayoría de las personas vienen comercializando (Barradas, 2020), y comprando estas monedas como mecanismos de inversión para el futuro o para sus negocios. (Carrera, Sánchez, \& Loza, 2020).

Asimismo, la revolución de las monedas digitales ha hecho que se supere el PIB en países como Suiza, Países Bajos, entre otros, movilizando más de 810 mil millones de US \$, desde el 2017 cuando nacen las monedas digitales, valorándose exponencialmente, pero que a partir de ese año ha ido en subida y descenso alcanzando e mediados de junio 
2019 cantidades capitalizables de 366 millones de dólares.

Hoy se puede comentar de este tipo de monedas sobre la volatilidad, métodos inversionistas, carencia de información, son puntos coherentes de información que requieren los inversionistas de hoy, por lo que se quiere decir que las inversiones en este tipo de monedas son muy riesgosas, cualquier inversionista deberá estar consciente del riesgo que pudiera surgir.

Hoy día existen más de 150 criptomonedas virtuales, y las que más demandan en el mercado son:

La primera fue Bitcoin, fue el ejemplo a seguir de todas las demás criptomonedas; creada por Satoshi Nakamoto en el 2009, su tecnología está basada en el blockchain

Es una moneda virtual, creada, para transferirla, depositarla, todo de forma virtual, nadie puede tener control sobre ella, está descentralizada, fuera del apoyo de bancos centrales y gobiernos, esto las caracteriza de forma digital, se le conoce como Btc., no tiene rivalidad, como característica además adiciona que su pago es descentralizado, no tiene intermediarios, es directa su transacción, son absolutamente anónimas, tiene un límite máximo hasta de 21 millones de Btc y no son embargables.

Todas las operaciones se realizan a través de una computadora, por eso dependen de un ordenador e internet; y para hacerse al uso de esta moneda, primero tiene que hacerse con una cartera virtual, instalada en su móvil o computador, cada una de ellas cuenta con una llave de dominio privado, de las que son utilizadas para firmas electrónicas, así que identifica la identidad del usuario y evita que se produzcan alteraciones.
Todas las operaciones de Btc son efectuadas en un registro público de blockchain, que es una red automatizada de ordenadores distribuidos alrededor del mundo, lo que implica que es una moneda que se descentralizó de cualquier banco central y no la controla ningún gobierno, esto implica que no la ampara ningún banco ni intermediario, además trata transacciones anónimas entre dos usuarios, libres de impuestos y comisiones.

De allí que el Btc se ha convertido en pagos recurrentes para transacciones de dudosa legalidad como el blanqueo de capitales, ello no implica que todas las demás criptomonedas operen legalmente. El mercado del Btc es muy volátil, porque está basado en la oferta y la demanda, calculada a través de un algoritmo que mide la cantidad de operaciones con este tipo de monedas; su valor va en escala.

Se pueden obtener Btc de diferentes maneras, a través de cajeros automáticos, cambiando dinero real por Btc a dólares, cajeros que iniciaron operaciones en Inglaterra y España, ya se encuentran en casi toda Europa.

También se afirma que se están imprimiendo billetes inteligentes de monedas virtuales Bitcoin (Montoya, 2020),que son comprables con la moneda actual, lo cual es tangible y práctico, seguro para realizar transacciones; pretendiéndose con esto cerrar el cerco de intangible a tangible; es decir la criptomoneda ya existe en billete físico, en denominaciones de 0,01 a 0,05 BTC, relativamente económicos, y realizados por la empresa Suiza Tangem, bajo la tecnología de Samsung; y los cuales se encuentran circulando en Singapur; la empresa Tangem fue sometida a una auditoría intensa y exhaustiva por parte de la empresa Kidelski Group para dar fe pública sobre la seguridad de los billetes. (Medina, 2020); (Lecaros, 2019); (Martínez, 2020) 


\section{6}

Ethereum, criptomoneda creada en el 2014 por Vitalik Buterin, encierra no solamente a su plataforma Ether, su principal finalidad es crear contratos inteligentes "Smarth Contracts".

Este tipo de contratos trabajan con plataformas informáticas inteligentes a través de blockchain, de forma descentralizada, con software de códigos para la ejecución de acuerdos entre los contratantes, que por lo general se incluye una transacción económica financiera, no tienen límite máximo.

Ripple: es una moneda que fue creada para establecer pagos digitalizados y transferencias entre fronteras de diferentes países, cuyas transacciones son instantáneas, evitando que pasen por intermediarios, se ahorra en costos, no generan problemáticas de escalada y trazabilidad en tiempo real.

Tether, (USDT) es una criptomoneda que se cotiza paralela al dólar, fue creada en el 2015 con la intención de no fluctuar en el cambio y su cotización que sea permanente; se conoce como "cripto/dólar" y entra con la combinando los dos mundos, con el objeto de comparar los atributos que brinda la plataforma digital blockchain como son la descentralización de la criptomoneda, confianza e irreversibilidad para compararse con todas las demás monedas en cuanto a ser seguras y de fácil aceptación.

La garantía en esta criptomoneda es que todas las transacciones están respaldadas con el mismo monto de dólares en sus cuentas de bancos. Lo cual con 1 USDT es un 1 USD\$, pues la característica principal es que es el precio de ella que la difiere de otras criptomonedas, es en su estabilidad.

Bitcoin Cash, es la criptomoneda muy similar al Btc, fue creada en el 2017, dispone de las mismas características que la moneda original que le aporta el blockchain, es contante, descentralizada, anónima, no se puede modificar; pero presenta incompatibilidad con la original, pues cada cual lleva su rumbo diferente.

La diferencia entre ambas reside en la capacidad que tiene el bloque. Esta nueva criptomoneda tiene capacidad de $8 \mathrm{Mb}$ y la primera de $1 \mathrm{Mb}$, lo que hace que la Btc cash incremente rápidamente más transacciones en un segundo. Hoy Btc cash permite máximo 7 operaciones en un segundo.

Leticoin (LTC) fue creada por Charlie Lee y es complementaria del Btc inicia en el 2011, tenía como finalidad ser complementaria del Btc, pero debido a los cambios a su plataforma resultó ser independiente totalmente, para hacer una comparación, se le denomina al Btc en la red patrón oro 2.0 y al Ltc, plata 2.0; su objeto inicial era la reducción del tiempo en las transacciones para confirmarlas y que participara cualquier persona en esta minería, pues posee la siguientes características diferenciadas con relación al Btc:

- Participación en la minería de datos, es mucho más fácil entrar en ella que la del Btc

- Escalabilidad: máximo de exitencia de Btc 21 millones, y Ltc tiene límite de 84 millones.

- Tiempo para las transacciones: 2,5 min. el Btc puede tardar 10 min.

Dogecoin, muy contraria a Btc tiene un bloque de 1 min., nace en el 2013 por Palmer y Markus, al igual que la PPC, no tiene cantidad de límite, y fue creada para pequeñas operaciones financieras.

Eos, inicia en el 2017 a través de la Oferta Inicial de la Moneda (ICO) es una criptomoneda diseñada para el desarrollo de actividades descentralizadas y con la capacidad para la descentralización de las Apps que existen. De forma tal que se amparan bajo la plataforma de blockchain. Y la EOS puede reprogramarse 
para descentralizarse, hay quienes opinan que puede cambiar revolucionariamente el sistema financiero.

Binance Coin (BNB) es una criptomoneda de "Exchange Binance", creada con el objetivo de que los usuarios realicen sus comercios a través de esta plataforma, y a los que tienen esta criptomoneda Binance coin aplica tarifas especiales de tipo comercial para rebajas y descuentos en el comercio de productos.

Esta moneda es un "token ERC20", estandarizado por Ethereum, usa blockchain y software de internt con códigos abiertos, admite intercambios con el resto de las monedas digitales para las transacciones comerciales de los productos.

Bitcoin Sv, es la división del Btc Cash, puesta en marcha en el 2018, sus siglas obedecen a "Bitcoin Satoschi's Vision", cuyos fundadores pretenden mantener "el protocolo original" Btc, de gran en escala, es estable e identifica problemas de; está desarrollada para que sirva de medios de pago por intermedio de operaciones rápidas y brinda seguridad.

Stellar, surge en 2014, sus creadores McCaleb y Mullenweg, es sinónima de Ripple, crear una red de operaciones internacionales de intercambio comercial de dinero; es perfecta para pagos pequeños, dada la rapidez de las transacciones, y bajas comisiones.

Lumens (XLM), es una moneda derivada de la plataforma de Stellar; actúa como intermediaria, brinda el facilismo entre plataformas digitales de criptomonedas para la realización de conversiones con el dólar y euro. Ejemplo, cuando la realización es de conversión de las criptomonedas al cambio con el valor del dólar o euro a lumens y luego de éstas a Btc.
Peer Coin (PPC), fue creada por Nadal y King, $\mathrm{y}$ es pionera en las pruebas de digitales de estado y de trabajo, esta red consume menor energía, por ello se creó de forma ilimitada para que superara las del Btc, estimado en 21 millones; PPC tiene un mercado actual que oscila en USD\$21.2 millones.

DCEP "Digital Currency Electronic Payment" es la primera moneda digital China, desde inicios del 2020 y paralizada por la pandemia, el país hace importantes experimentos para que esté respaldada por el Banco Central Chino, y se espera que para el 2022 la moneda sustituya al Yuan en papel y en moneda.

Blockchain: es una plataforma presenta importantes características para que el usuario pueda realizar las transacciones económicas y donde se apoyan las criptomonedas, estas son:

- Ahorra tiempo y costo en las operaciones de registro y controla la data, es una cadena de bloques que no permite que se registren duplicaciones.

- La seguridad que brinda es que no se pueda modificar nada, solamente añadir, o sea que no se permiten revertir cuestiones o dañar intencionalmente la plataforma.

- Transparencia: opera como si fuera el libro mayor contable donde se almacenarán las diferentes operaciones que se hagan, sólo se puede consultar, no se puede modificar tal operación, donde se garantiza la operación de forma eficiente.

- Velocidad en las operaciones, opera inmediatamente en el mismo tiempo, reduce riesgos de otras operaciones, en los cuales el compromiso para pagar demore días al ejecutarse, evita los riesgos de no pagar. 


\section{8}

Las desventajas de esta plataforma es la velocidad o escalabilidad, es lenta, aunque el tamaño de la base de datos crece rápidamente, hoy el tamaño del Btc es mucho más alto de $100 \mathrm{~GB}$, y no se puede utilizar para fines de usuarios domésticos.

Respecto a la regulación es incierta, porque no se conoce el respaldo que le den los Bancos Centrales europeos o de Norte América, respecto a la plataforma.

En cuanto a desventajas, en las criptomonedas, por esta parte, Krugman, Paul, Nobel economista, dice que el Btc borra 300 años de historia económica de materia monetaria; luego el "Lobo de Wall Street, Jordan Belfort" dice que "salga si no quiere perder todo su dinero", es decir salir de todo tipo de monedas; en España se habla que es una criptomoneda para lavar dinero.

Es algo muy diferente a lo que se utiliza en Colombia: Western Unión Pse, Paypal, se utiliza, los procesadores de pago tarjetas de Visa o Master Card, se utiliza un tercero para una transferencia internacional, el dinero se "toca físicamente", esta es una de las grandes diferencias con las transacciones de criptomonedas.

\section{Comportamiento económico en materia de inversiones en Colombia.}

Resulta imperioso hablar de la moneda colombiana virtual, el Triskel, que debiendo conocer su origen es propio de la cultura Celta que guarda relaciones sobre su simbología de tres espirales interconectados entre sí. (Díaz, 2018).

La moneda cuenta con el respaldo Finca Raíz, registro de transacciones, el usuario puede aceptar o rechazar la moneda, evitando que se reciban transacciones de dudoso origen; se habla de facilidad para realizar transacciones y de estabilidad, utilizable en diferentes pagos que requiera la persona hacer; pues maneja comisiones muy bajas del mercado, consultable en el Manual de Fenix Premium por internet, y ofrece alternativas de negocios, brinda descuentos para quienes comercialicen a través de ella, lista para realizar transacciones de una wallet a otra. (ESUMER, 2018); (Ruiz, Buitrago, \& Rodriguez, 2018); (Pacheco, 2016).

Con las criptomonedas, para entender este flagelo económico el Superintendente de Sociedades en Colombia, (Navarro, 2019), se da un comercio entre de multinivel, es legal, donde una gran cantidad de empresas se contratan entre sí en una gran red de ventas, para la promoción de mercancías y se prestan servicios; se les paga a los comisionistas y es rentable para incrementar las ventas. (Dorati, Ruda, \& González, 2019); (Catache, González, \& Pedroza, 2020); (Jiménez \& Santana, 2018).

Todas las monedas parecen brindar alto contenido de confiabilidad, son muchos los expositores que hablan de acogerse a este tipo de economía. (Mendoza, 2019). Hace años se dio un delito de estafa a escala nacional o mundial, con la famosa "pirámide" sólo lograba ganar o recoger el dinero el que estaba en la cúspide; hoy día este planteamiento está más vivo que nunca. (Salmerón, 2017); (Reza, Calderón, Sánchez, Macas, \& Corone, 2018).

Pero por esta parte, esta moneda virtual, como muchas otras pueden no ser confiables, porque existen otras monedas que se ofrecen por menor o mayor valor que no están todavía en el mercado. (Andrews \& Lara, 2020), (Vilarroig, 2018).

Porque, sobre invertir o no invertir en criptomonedas en Colombia, no está muy clara, existen una moneda ICO (que aún no están en el mercado), (García \& Rejas, 2020) es muy importante tomar decisiones exactas, algunas pueden resultar fraudulentas, 
ninguna criptomoneda cobra comisiones, como se pretende afirmar del a Triskel; por otra parte si se invierte en alguna moneda no aprobada y ésta cierra su página web, al invertir en este tipo de negocios no es confiable, perderá su inversión, porque la moneda no fue aprobada. (Giménez, María, \& Hernáez, 2018). Para ello se deben consultar las páginas sobre criptomonedas como la Coinshendule.com que brindan asesoría sobre este tipo de plataformas para que no se incremente más el delito de estafa. (Pérez, 2018); (Bedecarratz, 2018); (García \& Gonzalo, 2019); y (Nieto \& Hernández, 2018).

En Colombia el Bitcoin es famoso, es un fenómeno mundial, funciona como un blockchain (Bartolomé, 2020) y (Castellanos, 2017), que funciona solo en internet, de gran complejidad, las personas deben tener educación financiera y estar conscientes de los enormes riesgos que pueden incurrir en esta clase de inversiones porque esta moneda no está centralizada (López, 2018), no existe banco, no hay un país que la respalde, no existe un seguro para los depósitos (Méndez, 2018), no existe seguridad en caso de derrumbe, y no está respaldado por el Banco de la República, ni está emitido por ninguna autoridad financiera (Morten, 2017), eso no equivale a tener en físico: dólares, libras esterlinas o euros, entre otras monedas fuertes. (Martin \& Pons, 2019); (Terán \& Betti, 2018);

En Colombia no hay prohibición para comprar monedas virtuales, bitcoin por ejemplo o cualquier otra, pero debe estar la persona consciente del riesgo, no son divisas, ningún estado respalda esas operaciones; porque si el mercado ofrece tasas exorbitantes en alza, es un claro riesgo (Sánchez, 2018); invertir en operaciones bancarias a través de la banca tiene un seguro de depósito está respaldo a través de la Superintendencia de Operaciones
Financieras, que se le respalda hasta cierto monto en caso de haber problemas. (Giraldo, 2019); (Asto \& Villavicencio, 2019); (André, 2019); (Peña, 2017).

\section{Discusión}

Con esta revolución de la economía digital, en la que se pretende desplazar el papel moneda, conllevado a que la mayoría de las transacciones se realicen en forma virtual (Palma, 2020), a lo que ya los colombianos estaban siendo instruidos a través de realizar operaciones con tarjetas de débito o crédito, o la simple utilización de las plataformas bancarias; se complementa una nueva revolución en sus quehaceres, la tecnología digital de la moneda, (Morales \& Perea, 2018)

Asimismo, en países europeos la criptomoneda digital es una inversión, pero se pretende el desplazamiento del papel moneda, para dar cabida a una nueva moneda digital, tal es el caso del Bitcoin, que ha estado rodando desde el 2009 como moneda intangible, pero que hoy día ya están disponibles billetes en físico de Bitcoin en denominaciones de 0,01 y 0,05, circulando por Europa. (Moreno, Valencia, \& Soto, 2018)

En cuanto a la discusión dl objetivo específico uno, sobre el comportmaiento económico en materia de inversiones, se puede mencionar, por esta parte, la moneda colombiana, el Triskel representa una inversión, pero también una incertidumbre, porque el comportamiento económico en Colombia en cuestión de inversiones, como se dijo, no está muy claro con la moneda virtual en lo que respecta al Triskel o cualquier otra, pero una moneda sólida lo brinda la economía con el Bitcoin, aunque el inversor debe tener claro los riesgos que se le pudieran presentar a la hora de una caída fuerte, no tiene ningún respaldo. 
Respecto al análisis si las operaciones cambiarias en criptomonedas pretenden el desplazamiento del dólar; no está muy claro tampoco el desplazamiento del dólar, aunque para los inversionistas, todas, pero casi todas las inversiones las realizan en divisas, fijando como base el valor del dólar, cualquier importación o exportación de materia prima, insumos, o cualquier negociación se realiza es con la divisa norteamericana, en consecuencia es nada probable que se pueda dar como un desplazamiento de esta moneda, ya que es la moneda más fuerte del mercado y está respaldada por un país: Estados Unidos.

Con respecto al objetivo específico tres, sobre si las criptomonedas son confiables para las inversiones económicas de las empresas colombianas; es algo al azar, tiene sus márgenes de duda, porque:

Por otra parte, si para adquirir un bitcoin, es necesito invertir más de un dólar; una persona que cuente con grandes sumas millonarias en bitcoin, no tiene su economía asegurada (Cortez \& Tulcanaza, 2018), por más de que haya adquirido el papel de las criptomonedas en Singapur, porque a la hora de un desplome del Bitcoin nadie le va a respaldar la inversión; únicamente tiene respaldo financiero, cuando se encuentre la moneda aparada por un banco, Banco de la República, por ejemplo, pero este banco de Colombia, ni ningún otro ha respaldado criptomoneda alguna. (Agila, 2019)

Por otra parte, existen muchos inversionistas, o comerciantes, que prefieren invertir en monedad virtuales, pero sucede que estas inversiones, cada día cambian su valor en aumento, con altos rendimientos, es algo que puede ser ficticio porque puede el comerciante: ganar mucho o perderlo todo; haría falta que los comerciantes estudiaran economía y finanzas sobre las criptomonedas específicamente para que sepan a qué atenerse a la hora de un embarque. (Morales \& Quimbiulco, 2019);

\section{Conclusiones}

Las criptomonedas en Colombia hay iniciativas de regulación; porque la Superintendencia Financiera está enviando aletas sobre estos planes de inversión.

El problema de adaptación es la protección, la criptomoneda no tienen marco de regulación en el país; el país tiene un proyecto de ley pero no se ha aprobado hasta el día de hoy.

El blockchain es una tecnología que permite el acceso a productos y servicios sin necesidad de entidades intermediarias. Antes se necesitaba a ciertas entidades para el respaldo del dinero, ahora hay empresas acumulan datos y también hay tenido fuertes caídas como la bolsa de 2008; ahora estas tecnologías se apoyan en mercados para realizar transacciones de persona a persona sin necesitar de las tecnologías financieras, mucho más eficientes y rápidas.

Muchas personas en Colombia están comprando monedas, y esto es una forma a nivel mundial, es una zona color gris, que ha tenido alarmas, que se inició con el lavado de dinero, pero que hoy tiene otros fines, las propias criptomonedas; cualquier persona en Colombia puede tener su propia moneda; se dice que hasta el mismo jugador Colombiano James, ha adquirido su propia criptomoneda; es decir cualquier persona puede tener una, pero el éxito de ella es generar mucha confianza para que la gente invierta en ella.

Es beneficiosa la regulación de las criptomonedas porque al tener el respaldo del blockchain, puede eliminar el lavado de dinero, también el tema de los impuestos; y 
para ello es necesario registrar y poner reglas claras que confirmen quienes son los actores que pueden participar en las operaciones de esas criptomonedas y cómo han de hacerlo, porque definir el porcentaje por recursos para transacciones es un tema que tiene que fijar, quien respalde esta monetización.

Se ha puesto el tema de la monetización del futuro ante el debate de la opinión pública y ante el Congreso, y éste es quien tiene la facultad legal y la responsabilidad ante lo que está sucediendo en el país.

Esto es un tema que no es novedoso, una persona particular para adquirir un vehículo puede hacerlo con Bitcoin directamente a USA, lo cancela desde Colombia y recibe el vehículo aquí; pero los comerciantes pueden verse afectados porque bajarían sus ventas por los costos elevados de transporte y otros gastos de mantenimiento.

También se puede evidenciar que las plataformas son pseudo-anónimas, se podrían prestar para lavado de dinero, de activos, financiamiento terrorista, entre otros aspectos ilegales. Así la tecnología es neutral, y la criptomoneda no es algo que haya que atacar, porque su uso lo realizan las personas no la tecnología.

Igualmente a nivel mundial se han presentado escándalos sobre lavados de activos en bancos europeos, hoy el monto total de las criptomonedas es de 200 billones de dólares. Existe mucha publicidad para el lavado de activos, pero es algo que día a día se sigue regulando para evitar este tipo de fraudes y que la criptomoneda sea confiable.

\section{Referencias}

Agila, S. R. (04 de 2019). El Bitcoin: Crecimiento y desarrollo de la criptomoneda y su inclusión como alternativa de pago y medio de intercambio en la economía ecuatoriana, período: 20142018. Guayaqiol. Obtenido de http://repositorio. ug.edu.ec/handle/redug/40599

André, M. E. (2019). Criptomonedas, Signos de Valor y Liberación Económica en América Latina. revista Otraeconomía, 12(21), 32-53. Obtenido de https://revistaotraeconomia.org/ index.php/otraeconomia/article/view/14798

Andrews, S. K., \& Lara, G. G. (2020). Las monedas complementarias y la economía de los individuos: Un estudio en el Municipio de Santiago de Querétaro. Sinapsis. Revista de investigaciones de la Institución Universitaria EAM, 48-68. Obtenido de https://dialnet. unirioja.es/descarga/articulo/7571138.pdf

Asto, P. N., \& Villavicencio, F. M. (2019). ¿Las criptomonedas deben ser consideradas dinero? Obtenido de https://repositorioacademico.upc. edu.pe/handle/10757/626341

Barradas, G. J. (2020). Las Criptomonedas: Un Desafío Empresarial. Aula Virtual, 70-80. Obtenido de http://aulavirtual.web.ve/revista/ ojs/index.php/aulavirtual/article/view/18

Barrutia, B. I., Urquizo, M. J., \& Acevedo, S. I. (2019). Criptomonedas y blockchain en el turismo como estrategia para reducir la pobreza. Retos. Revista de Ciencias de la Administración y Economía., 287-302. Obtenido de http:// scielo.senescyt.gob.ec/scielo.php? script $=$ sci arttext\&pid=S1390-86182019000200287

Bartolomé, P. A. (2020). Cambiando el futuro: "Blockchain" y Educación". Pixel-Bit. Revista de Medios y Educación, 241-258. Obtenido de https://recyt.fecyt.es/index.php/pixel/article/ view/82546

Bedecarratz, S. F. (2018). Riesgos delictivos de las monedas virtuales: Nuevos desafíos para el derecho penal. Revista chilena de derecho y 


\section{2}

tecnología, 79-105.

Caballero, M. J. (2019). Criptomonedas, blockchain y contratos inteligentes. Obtenido de https:// bdigital.uexternado.edu.co/handle/001/2592

Carrera, L. ,., Sánchez, L. J., \& Loza, T. A. (2020). El uso de las criptomonedas como nueva forma de pago en la economía mundial. FIPCAEC, 210-223. Obtenido de https://fipcaec.com/index. php/fipcaec/article/view/228/380

Castellanos, E. (2017). CRIPTOMONEDAS, BLOCKCHAIN Y UNA NUEVA VISIÓN DEL MUNDO. Colombia. Obtenido de http://codeamed.com.co/wp-content/ uploads/2018/05/Erick-CastellanosCriptomonedas-Blockchain-y-una-nuevavisi $\% \mathrm{C} 3 \% \mathrm{~B} 3 n$-del-mundo.pdf

Catache, M. M., González, T. S., \& Pedroza, C. G. (2020). Conocimientos y hábitos de inversiones en criptomonedas: Comparación entre Generación X y Generación Y . Revista Relayn, 26-34. Obtenido de https://iquatroeditores.com/ revista/index.php/relayn/article/view/284/164

Cortez, A., \& Tulcanaza, A. (2018). Bitcoin: $\mathrm{Su}$ Influencia En El Mundo Global Y Su Relación Con El Mercado De Valores. Revista Chakiñan de Ciencias Sociales y Humanidades(5), 1-10. Obtenido de http:// scielo.senescyt.gob.ec/scielo.php?script $=$ sci arttext\&pid=S2550-67222018000100054

Díaz, d. B. (2018). El devenir de Bitcoin : aspectos y consideraciones a tener en cuenta en lo que a su futuro se refiere. Obtenido de https://repositorio. comillas.edu/xmlui/handle/11531/19993

Dorati, J., Ruda, R., \& González, I. (2019). Panorama Global De Las Criptomonedas Y Su Desarrollo. Panorama Global, 129-144. Obtenido de http:// revistas.ulatina.edu.pa/index.php/genteclave/ article/view/122/135
ESUMER. (2018). Observatorio de Tendencias Futuro $360^{\circ}$ \& Institución Universitaria Esumer. Criptomonedas y nuevos medios de intercambio virtual. Obtenido de http://repositorio.esumer. edu.co/handle/esumer/1908

Fernando, R. C., \& González, M. R. (2018). El Dinero Electrónico Su Participación En La Economia. Revista Observatorio de la Economía Latinoamericana. Obtenido de https://www. eumed.net/rev/oel/2018/06/dinero-electronicoeconomia.zip

García, A., \& Gonzalo, R. (2019). Bitcoin, La regulación legal de las monedas virtuales. Obtenido de https://repositorio.uesiglo21. edu.ar/bitstream/handle/ues21/16392/ A L B A \% 20 GA R C I A \% 20 RODRIGO. pdf?sequence $=1 \&$ isAllowed $=\mathrm{y}$

García, R. L., \& Rejas, M. R. (2020). Balance del régimen jurídico-económico de las ICOs en la financiación de las PYMES. Derecho y Cambio Social, 489-501. Obtenido de https://dialnet. unirioja.es/servlet/articulo?codigo $=7525026$

Giménez, N., María, \& Hernáez, M. J. (2018). Monedas Virtuales Y Locales: Las Paramonedas, ¿Nuevas Formas De Dinero? Revista de estabilidad financiera, 1-20. Obtenido de https://repositorio.bde. es/bitstream/123456789/11245/1/Monedas_ virtuales_y_locales_las_paramonedas.pdf

Giraldo, V. L. (2019). Realidad contable de las criptomonedas en Latinoamérica. Obtenido de https://ojs.tdea.edu.co/index.php/agora/article/ view/737/883

Jiménez, G. C. (2020). ¿El Fin Del Dinero Físico? San Cristóbal de La Laguna. Obtenido de https://riull.ull.es/xmlui/bitstream/ handle/915/19373/\%C2\%BFE1\%20fin \%20 del $\% 20$ dinero $\% 20$ fisico.pdf?sequence $=1$ 
Jiménez, M. E., \& Santana, C. J. (2018). Propuesta De Un Índice De Mercado Para Criptomonedas: Crypto-10. Revista Universitaria Ruta,, 20(1), 49-67. Obtenido de https://revistas.userena.cl/ index.php/ruta/article/view/1084

Lecaros, S. P. (2019). Tributación de criptomonedas. Obtenido de http://repositorio.uchile.cl/ handle/2250/170176?show=full

López, R. B. (06 de 2018). Estudio de tecnologías Bitcoin y Blockchain. Barcelona. Obtenido de http:/openaccess.uoc.edu/webapps/o2/ handle/10609/81739

Martin, T. A., \& Pons, C. M. (2019). Bitcoin: ¿La moneda del futuro? Moneda electrònica Bitcoin. Quaderns IAFI, 127-158. Obtenido de http:// diposit.ub.edu/dspace/handle/2445/165861

Martínez, C. J. (2020). Valor y futuro de las criptomonedas: Análisis crítico. Obtenido de https://uvadoc.uva.es/handle/10324/42041

Mecheba, M. J. (2016). Bitcoin, ¿la moneda del futuro? La Coruña. Obtenido de https://ruc.udc. es/dspace/handle/2183/18055

Medina, A. A. (2020). Las Criptomonedas: Especial Referencia Al Bitcoin. Obtenido de https:// riull.ull.es/xmlui/bitstream/handle/915/21456/ Las\%20Criptomonedas\%20Especial\%20 Referencia\%20al\%20Bitcoin.pdf?sequence $=1$

Melguizo, M. C. (2020). Ventajas Y Desventajas De Las Criptomonedas En La Economía Colombiana. Bogotá. Obtenido de https://repository.unimilitar. edu.co/bitstream/handle/10654/35279/ M e $1 \mathrm{~g} \mathrm{u}$ i z o M or e n o C a r 1 os 2019. pdf?sequence $=1 \&$ isAllowed $=y$

Méndez, B. M. (2018). Responsabilidad del estado frente a la circulación de monedas electrónicas en Colombia: el bitcoin. Obtenido de https:// repository.ucatolica.edu.co/handle/10983/22577

Mendoza, T. J. (2019). Innovación disruptiva de las criptomonedas para la sociedad y el comercio electrónico. Obtenido de http://rua.ua.es/ dspace/handle/10045/95528

Montoya, R. J. (2020). El bitcoin una innovación financiera. Obtenido de https://repository. upb.edu.co/handle/20.500.11912/5935?localeattribute $=\mathrm{es}$

Morales, C. A., \& Perea, M. L. (21 de 09 de 2018). El Bitcoin en el Sistema Financiero. Pereira. Obtenido de http://repositorio.unilibrepereira. edu.co:8080/pereira/handle/123456789/1241

Morales, M. M., \& Quimbiulco, C. R. (2019). Plataformas colaborativas como medio de promoción e impulso para el uso de Bitcoin. Quito. Obtenido de http://www.dspace.uce.edu. ec/handle/25000/19362

Moreno, R. B., Valencia, N., \& Soto, F. (2018). Criptomonedas como alternativa de inversión, riesgos, regulación y posibilidad de monetización en Colombia: el Bitcoin. Obtenido de https://expeditiorepositorio.utadeo.edu.co/ handle/20.500.12010/4746

Morten, B. R. (2017). Criptomonedas De Bancos. Informe Trimestral de bpi, septiembre de 2017, 99-119. Obtenido de https://www.cemla.org/ PDF/boletin/PUB_BOL_LXIV-01-03.pdf

Navarro, C. F. (2019). Criptomonedas (en especial, bitcóin) y blanqueo de dinero. Revista Electrónica de Ciencia Penal y Criminología, 1-45. Obtenido de https://accedacris.ulpgc.es/ handle/10553/74463

Nieto, G. M., \& Hernández, M. J. (2018). Monedas virtuales y locales las paramonedas ¿Nuevas formas de dinero? Revista de Estabilidad Financiera. Obtenido de https://repositorio. 
bde.es/bitstream/123456789/11245/1/Monedas virtuales_y_locales_las_paramonedas.pdf

Pacheco, J. M. (2016). Criptodivisas: Del Bitcoin Al Mufg.El Potencial De La Tecnología Blockchain. Revista CESCO de Derecho de Consumo, 6-15. Obtenido de https://ruiderae. revista.uclm.es/index.php/cesco/article/ view/1180/952

Palma, C. M. (2020). El bitcoin: Comprendiendo el Mercado. Revista Científica Apuntes De Economía Y Sociedad,, 1, 9-24. Obtenido de http://revistas.unanleon.edu.ni/index.php/ apunteseconomiaysociedad/article/view/voll-12020-arto2

Peña, T. H. (2017). Un viaje por el pasado, presente y futuro del dinero virtual. Revista Tecnología, investigación y academica, 6(1), 67-71. Obtenido de https://revistas.udistrital.edu.co/index.php/ tia/article/view/12115/pdf

Pérez, B. B. (2018). La Administración tributaria frente al anonimato de las criptomonedas: la seudonimia del Bitcoin. Documentos de Trabajo del Instituto de Estudios Fiscales, 149-161. Obtenido de http://rua.ua.es/dspace/ handle/10045/83967

Restrepo, J. M., \& Ocampo, M. (2019). Las Criptomonedas: Ventajas Y Desventajas Para La Economía. Medellín. Obtenido de https://dspace.tdea.edu.co/bitstream/handle/ tda/561/LAS\%20CRIPTOMONEDAS\%20 VENTAJAS\%20Y\%20DESVENTAJAS. pdf?sequence $=1 \&$ isAllowed $=y$

Reza, s. L., Calderón, R. P., Sánchez, F., Macas, G., \& Corone, M. (2018). El Dinero Electrónico En El Ecuador, Su Uso En Escolares. Revista Observatorio de la Economía Latinoamericana, 1 - 10. Obtenido de https://www.eumed.net/rev/ oel/2018/04/dinero-electronico-ecuador.html
Ruiz, C. M., Buitrago, A. C., \& Rodriguez, D. S. (2018). Impacto de las criptomonedas en la economia colombiana en el año 2017 en la ciudad de Medellin. Medellín. Obtenido de https://dspace.tdea.edu.co/handle/tda/399

Salmerón, M. E. (2017). Necesaria regulación legal del Bitcoin en España. Revista de Derecho Civil, 293-297. Obtenido de https://dialnet. unirioja.es/servlet/articulo?codigo $=6267530$

Sánchez, L. R. (2018). Análisis teórico-práctico de la moneda virtual Bitcoin y sus implicaciones económicas. Obtenido de https://riull.ull.es/ xmlui/bitstream/handle/915/9266/Analisis\%20 teorico-practico $\% 20 \mathrm{de} \% 201 \mathrm{a} \% 20$ moneda $\% 20$ virtual $\% 20$ Bitcoin $\% 20$ y $\% 20$ sus $\% 20$ implicaciones $\% 20$ economicas.pdf?sequence $=1$

Terán, N. I., \& Betti, M. G. (2018). La Moneda Virtual Como Medio De Pago En El Comercio Electrónico. Caso Bitcoin. Obtenido de https:// core.ac.uk/download/pdf/232025863.pdf

Vilarroig, M. R. (2018). Tributación de criptomonedas. Revista de Economía Balance, 15-20. Obtenido de http://repositori.uji.es/ xmlui/bitstream/handle/10234/181070/61565. pdf?sequence $=1 \&$ isAllowed $=y$ 